\title{
Integrative genomic analysis for the functional roles of ITPKC in bone mineral density
}

\section{Hsing Fang Lu ${ }^{1}$, Henry Sung-Ching Wong ${ }^{1,2}$, Yu-Wen Hsu ${ }^{3}$, Er-Chieh Cho ${ }^{1}$, Kuo-Sheng Hung ${ }^{4}$, Wei- Chiao Chang ${ }^{1,2,5,6}$}

${ }^{I}$ School of Pharmacy, Taipei medical university, Taiwan, ${ }^{2}$ Master Program for Clinical Pharmacogenomics and Pharmacoproteomics, School of Pharmacy, Taipei Medical University, Taiwan, ${ }^{3}$ The Ph. D. Program for Translational Medicine, College of Medical Science and Technology, Taipei Medical University and Academia Sinica, Taiwan, ${ }^{4}$ Department of Neurosurgery, Taipei Medical University-Wan Fang Hospital, Taiwan, ${ }^{5}$ Department of Pharmacy, Taipei Medical University-Wanfang Hospital, Taiwan, ${ }^{6}$ Center for Biomarkers and Biotech Drugs, Kaohsiung Medical University, Taiwan

SOC channel is the main calcium signal channel in $\mathrm{T}$ cells and osteoclast cells. Previous studies indicate that early activation of osteoclast relies on calcium entry through store-operated calcium entry (SOCE). Knockdown the expression of STIM1 in osteoclast will diminish their differentiation. Orai1-/- mice also shows significantly decrease in cortical ossification. Inositol-trisphosphate 3-kinase C (ITPKC) is a negative regulator of the SOC channel. However, the relation between STIM1, Orai1, ITPKC and bone mineral density (BMD) are still unclear. In order to investigate the plausible association with SOCE genes and BMD, we conducted a meta-analysis of genes expression and BMD using publicly available GEO database. We further recruited a total of 1044 subjects and tested the association between the polymorphism of these genes and BMD. Clinical information (including age, sex, and BMI) was collected and used for the analysis. Our results indicated that the gene expression of ITPKC was significantly associated with BMD. Furthermore, we found that one SNPs (rs2607420) of ITPKC was significantly associated with lumbar spine BMD. Through bioinformatics analysis, rs2607420 was a candidate that involved in the regulation of ITPKC expression. Our findings suggest that ITPKC is a susceptibility gene for BMD, and rs2607420 may play an important role in the regulation of this gene. 